EPJ Web of Conferences 64,06011 (2014)

DOI: $10.1051 /$ epjconf/ 20146406011

(C) Owned by the authors, published by EDP Sciences, 2014

\title{
NUSTAR detection of 4s Hard X-ray Lags from the Accreting Pulsar GS 0834-430
}

\author{
Matteo Bachetti ${ }^{1,2, a}$, Hiromasa Miyasaka ${ }^{3}$, Fiona Harrison ${ }^{3}$, Felix Fürst ${ }^{3}$, Didier Barret ${ }^{1,2}$, Eric \\ C. Bellm ${ }^{3}$, Steven E. Boggs ${ }^{4}$, Deepto Chakrabarty ${ }^{5}$, Jerome Chenevez ${ }^{6}$, Finn E. Christensen ${ }^{6}$, \\ William W. Craig ${ }^{4,7}$, Brian W. Grefenstette ${ }^{3}$, Charles J. Hailey ${ }^{8}$, Kristin K. Madsen ${ }^{3}$, Lorenzo \\ Natalucci ${ }^{9}$, Katja Pottschmidt ${ }^{10}$, Daniel Stern ${ }^{11}$, John A. Tomsick ${ }^{4}$, Dominic J. Walton ${ }^{3}$, Jörn \\ Wilms $^{12}$, and William Zhang ${ }^{13}$ \\ ${ }^{1}$ Université de Toulouse; UPS-OMP; IRAP; Toulouse, France \\ ${ }^{2}$ CNRS; Institut de Recherche en Astrophysique et Planétologie; 9 Av. colonel Roche, BP 44346, F-31028 \\ Toulouse cedex 4, France \\ ${ }^{3}$ Cahill Center for Astronomy and Astrophysics, California Institute of Technology, Pasadena, CA 91125 \\ ${ }^{4}$ Space Sciences Laboratory, University of California, Berkeley, CA 94720, USA \\ ${ }^{5}$ Kavli Institute for Astrophysics and Space Research, Massachusetts Institute of Technology, Cambridge, \\ MA 02139 \\ ${ }^{6}$ DTU Space, National Space Institute, Technical University of Denmark, Elektrovej 327, DK-2800 Lyngby, \\ Denmark \\ ${ }^{7}$ Lawrence Livermore National Laboratory, Livermore, CA 94550, USA \\ ${ }^{8}$ Columbia Astrophysics Laboratory, Columbia University, New York, NY 10027, USA \\ ${ }^{9}$ Istituto di Astrofisica e Planetologia Spaziali, INAF, Via Fosso del Cavaliere 100, Roma I-00133, Italy \\ ${ }^{10}$ CRESST, UMBC, and NASA GSFC, Code 661, Greenbelt, MD 20771, USA \\ ${ }^{11}$ Jet Propulsion Laboratory, California Institute of Technology, Pasadena, CA 91109, USA \\ ${ }^{12}$ Dr. Karl-Remeis-Sternwarte and ECAP, Sternwartstr. 7, 96049 Bamberg, Germany \\ ${ }^{13}$ NASA Goddard Space Flight Center, Greenbelt, MD 20771, USA
}

\begin{abstract}
The NUSTAR hard X-ray telescope observed the transient Be/X-ray binary GS 0834-430 during its 2012 outburst. The source is detected between $3-79 \mathrm{keV}$ with high statistical significance, and we were able to perform very accurate spectral and timing analysis. The phase-averaged spectrum is consistent with that observed in many other magnetized accreting pulsars. We fail to detect cyclotron resonance scattering features in either phase-averaged nor phase-resolved spectra that would allow us to constrain the pulsar's magnetic field. We detect a pulse period of $\sim 12.29 \mathrm{~s}$ in all energy bands. The pulse profile can be modeled with a double Gaussian and shows a strong and smooth hard lag of up to 0.3 cycles in phase, or about $4 \mathrm{~s}$ between the pulse at $\sim 3$ and $\gtrsim 30 \mathrm{keV}$. This is the first report of such a strong lag in high-mass X-ray binary (HMXB) pulsars. Previously reported lags have been significantly smaller in phase and restricted to low-energies $(\mathrm{E}<10 \mathrm{keV})$. We investigate the possible mechanisms that might produce such lags. We find the most likely explanation for this effect to be a complex beam geometry.
\end{abstract}

a e-mail: matteo.bachetti@irap.omp.eu

This is an Open Access article distributed under the terms of the Creative Commons Attribution License 2.0, which permits unrestricted use, distribution, and reproduction in any medium, provided the original work is properly cited. 

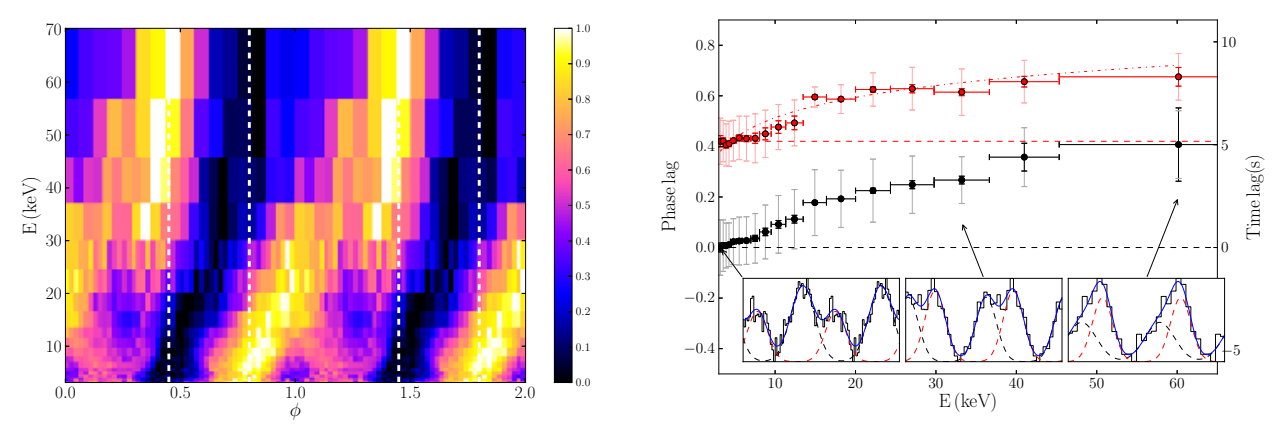

Figure 1. (Left) Evolution of the pulse profile with energy. Starting from an energy resolution of $\sim 0.4 \mathrm{keV}$ and 48 phase bins, we averaged over an increasing number of bins at higher energies, following approximately a geometrical progression both in phase and in energy in order to attain a better $\mathrm{S} / \mathrm{N}$. Each profile is backgroundsubtracted and normalized from 0 to 1 . This normalized rate is color-coded, ranging from the lowest count-rates in black to the highest in white. The profiles are repeated twice in phase for clarity. The white dashed lines indicate roughly the phase of the peak and the dip, respectively, at the softest energies to guide the eye. The minimum and the peak of the profiles are shifting in phase with energy, showing a clear hard lag. (Right) Phase lags of the two peaks, as obtained by fitting each rebinned profile with two independent Gaussians, relative to the phase of the first peak in the lowest energy band. Black is the main peak, red the secondary. Full-color error bars are $1-\sigma$ uncertainties, while light-colored error bars indicate the HWHM of the Gaussians. Dashed lines show the phase of the two peaks in the lowest energy band. The dash-dotted line indicates the fit of a Comptonization lag model to the secondary peak lags (see the details in [6]). The insets show the fit at $3 \mathrm{keV}$, at $\sim 35 \mathrm{keV}$ where the secondary peak becomes dominant, and at $\sim 60$ respectively.

\section{NUSTAR observation of GS $0834-430$}

GS 0834-430 is an accreting Pulsar discovered in 1990 [1]. It is an example of Be/X-ray binary. It has a spin period of $\sim 13 \mathrm{~s}$, an orbital period of $108 \mathrm{~d}$ with a wide orbit $\left(a_{x} \sin i \sim 128 l-s\right)$ [2] around a B0-2 V-IIIe companion [3]. At the peak of its latest outburst [4], on UT 2012 July 11, the source was observed by NuSTAR [5] for about $31 \mathrm{ks}$. In these pointings, at $10 \mathrm{keV}$ the source is $10^{3}$ times brighter than the background, and at $79 \mathrm{keV}$ the source is still 10 times above the background. We could perform very high quality timing and spectral analysis up to $79 \mathrm{keV}$.

The pulse profile is detected significantly up to $\sim 60 \mathrm{keV}$. The energy-resolved pulse profile shows clear phase lags between the pulse profiles at different energies. The shift is about 0.3 cycles in phase, or $4 \mathrm{~s}$ in time, between 3 and $30 \mathrm{keV}$. Fitting the profile with a double Gaussian, the two components seem to move together, almost linearly at first, then saturating above $20 \mathrm{keV}$ (Fig. 1). The primary peak dominates at low energy, then the second becomes the stronger one above $30 \mathrm{keV}$.

The source spectrum varies considerably with pulse phase. We use an NPEX model to characterize the spectrum in 10 phase bins (Fig. 1) of similar hardness ratio. The model fits adequately the spectrum for all phase bins, and there are no signatures that suggest the presence of a cyclotron line. A weak $(\mathrm{EW} \sim 40 \mathrm{eV})$ iron line is present, mildly anti-correlated with flux. We find no evidence of cyclotron resonance scattering features (CRSF), often observed in accreting pulsars and yielding an approximate measure of the magnetic field in these sources [see 7, for a review]. 


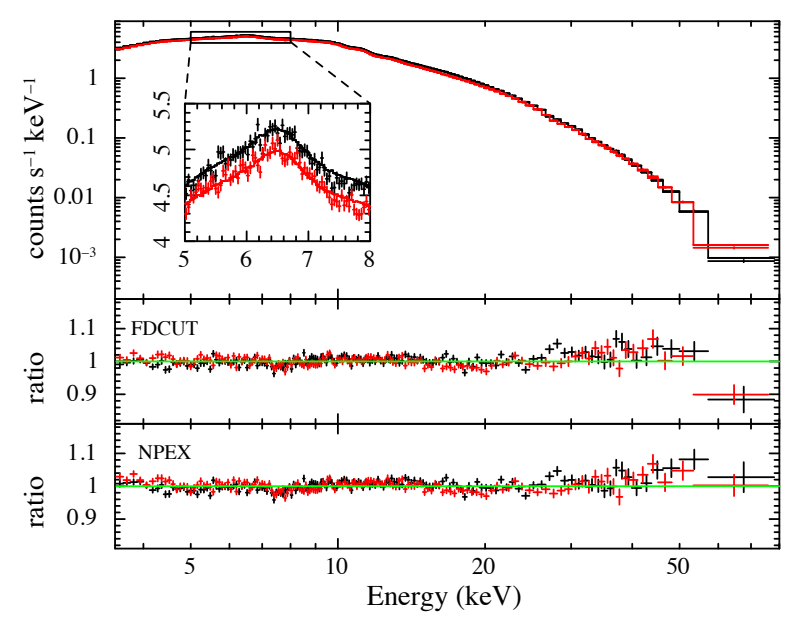

Figure 2. Phase-averaged spectrum fit using the FDCUT continuum model. Black points represent data from module FPMA, red from FPMB. The top panel shows the counts spectrum, with the data-to-model ratio for FDCUT and NPEX shown on the middle and bottom panels respectively. The region around the iron line is shown in the inset.

\section{Discussion}

Time lags are observed in many accreting sources [see 8 , for a review]. They can be produced through delays due, e.g., to Comptonization of the signal [e.g. 9], reflection from a medium far from the source [e.g. 10, 11], relativistic effects [e.g. 12], or propagation of signals in a disk [e.g. 13]. But 4s time lags are very difficult to reconcile with the most common geometries proposed for lag production (see the full discussion in [6]).

A CRSF can also produce a similar effect as the one we observe, producing an apparent delay in the spectral emission due to the different contributions of the feature in the various phases of the pulse profile [e.g. 14], but we do not find evidence for CRSFs in the spectrum in the relevant bands.

Similarly, it is possible that the effect is produced by the different emission components coming from the different regions of the accretion column. If the source has a luminosity between $\sim 10^{37} \mathrm{erg} \mathrm{s}^{-1}$, as is the case for GS 0834-430 during this outburst, calculations show that a Compton shock is formed in the accretion column, but that the luminosity is not sufficient to produce a radiation dominated shock, which would decelerate the infalling material high above the neutron star surface [15]. In this intermediate regime, a complex beam profile is therefore produced: radiation, after being upscattered to high energies by inverse Compton scattering inside the accretion column, emerges through the walls of the accretion column (fan beam), as well as along the magnetic field lines (pencil beam). The fan and pencil beam components can in principle have different spectral properties. Given the right geometry, this could create the phase-shift effect seen in the NUSTAR data through a gradual mixing of a softer and harder continuum. These processes are complicated and strongly dependent on the single source physical structure, and this might explain why only this source shows such strong lags.

If this is the case, our observation represents a very interesting step in the understanding of these processes. More details can be found in [6].

\section{References}

[1] R. Sunyaev, IAU Circ. 5122, 2 (1990)

[2] C.A. Wilson, M.H. Finger, B.A. Harmon, D.M. Scott, R.B. Wilson, L. Bildsten, D. Chakrabarty, T.A. Prince, ApJ 479, 388 (1997) 

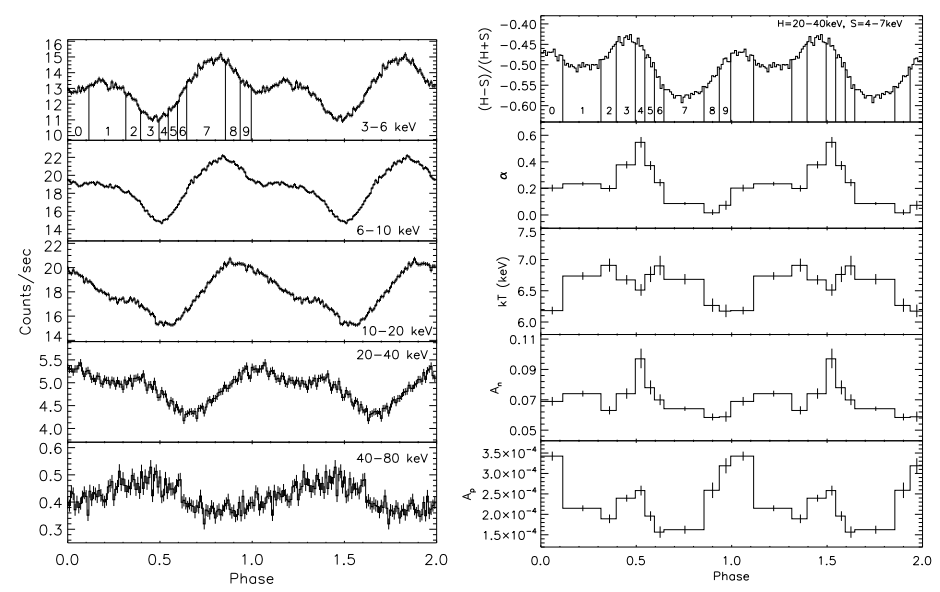

Figure 3. (Left) The pulse profile in four different energy bands. A strong dependence with energy is clearly visible. Intervals in the top pulse profile show the phases used for phase-resolved spectroscopy. (Right) Hardness ratio between the $20-40 \mathrm{keV}$ and $4-7 \mathrm{keV}$ bands as a function of pulse phase (top panel). Intervals have been chosen based on evolution of the hardness ration to fit NPEX model parameters. The lower three panels show the evolution of fit parameters as a function of pulse phase, i.e., the photon index of the negative powerlaw $(\alpha)$, the cutoff energy $k T$, the normalization of the negative powerlaw component $A_{n}$, and the normalization of the positive powerlaw component $A_{p}$. The pulse is repeated once for clarity.

[3] G.L. Israel, S. Covino, S. Campana, V.F. Polcaro, P. Roche, L. Stella, A. di Paola, D. Lazzati, S. Mereghetti, E. Giallongo et al., MNRAS 314, 87 (2000)

[4] S.P. Drave, V. Sguera, A.J. Bird, M. Goossens, L. Sidoli, A. Bazzano, M. Fiocchi, The Astronomer's Telegram 4218, 1 (2012)

[5] F.A. Harrison, W.W. Craig, F.E. Christensen, C.J. Hailey, W.W. Zhang, S.E. Boggs, D. Stern, W.R. Cook, K. Forster, P. Giommi et al., ApJ 770, 103 (2013)

[6] H. Miyasaka, M. Bachetti, F.A. Harrison, F. Fürst, D. Barret, E.C. Bellm, S.E. Boggs, D. Chakrabarty, J. Chenevez, F.E. Christensen et al., ApJ 775, 65 (2013)

[7] W. Coburn, P. Kretschmar, I. Kreykenbohm, V.A. McBride, R.E. Rothschild, R. Staubert, J. Wilms, ASR 38, 2747 (2006)

[8] J. Poutanen, ASR 28, 267 (2001)

[9] E.P. Liang, P.L. Nolan, SSRv 38, 353 (1984)

[10] M.M. Basko, R.A. Sunyaev, L.G. Titarchuk, A\&A 31, 249 (1974)

[11] I.M. George, A.C. Fabian, MNRAS 249, 352 (1991)

[12] W. Cui, E.H. Morgan, L.G. Titarchuk, ApJL 504, L27 (1998)

[13] Y.E. Lyubarskii, MNRAS 292, 679 (1997)

[14] C. Ferrigno, M. Falanga, E. Bozzo, P.A. Becker, D. Klochkov, A. Santangelo, A\&A 532, 76 (2011)

[15] P.A. Becker, D. Klochkov, G. Schönherr, O. Nishimura, C. Ferrigno, I. Caballero, P. Kretschmar, M.T. Wolff, J. Wilms, R. Staubert, A\&A 544, 123 (2012) 